\title{
An introduction to the IAAT ultraviolet MCP detector development
}

\author{
S. Hermanutz ${ }^{1}$, J. Barnstedt ${ }^{1}$, S. Diebold ${ }^{1}$, H.R. Elsener ${ }^{2}$, C. Kalkuhl ${ }^{1}$, N. Kappelmann ${ }^{1}$, \\ M. Pfeifer ${ }^{1}$, T. Schanz ${ }^{1}$, K. Werner ${ }^{1}$ \\ ${ }^{1}$ Institute for Astronomy and Astrophysics (IAAT), Eberhard Karls University Tübingen \\ ${ }^{2}$ Empa, Swiss Federal Laboratories for Materials Science and Technology, Dübendorf
}

\begin{abstract}
Observations of ultraviolet light is the key to understand high temperature processes in the universe like hot plasma, accretion processes or illuminated protoplanetary discs around UV sources. Furthermore these observation contribute to major cosmological questions, like the distribution of baryonic matter or the formation of the milky way, as pointed out by Gomez de Castro et al. ${ }^{1}$ Driven by the idea to participate in the Russian World Space Observatory we started to develop a position sensitive micro channel plate detector (MCP) for spectroscopy in the range of $160 \mathrm{~nm}$ to $300 \mathrm{~nm}$. Although we are not part of this project we still build a MCP detector prototype. In this paper we will present the general design of the detector and mainly focus on the aspect of our photocathode, while the electronics will be explained in more detail in the paper "Characterisation of low power readout electronics for a UV microchannel plate detector with cross-strip readout" (Paper number 9144-116) by Marc Pfeifer.
\end{abstract}

Keywords: micro channel plate detector, caesium telluride photocathodes

\section{THE MCP DETECTORS DESIGN}

The Institute of Astronomy and Astrophysics Tübingen has a long tradition in developing ultraviolet sensitive detectors and based on the heritage of the 1990's flight of ORFEUS we develop a position sensitive, solarblind, photon counting micro channel plate detector. Even if there is an ongoing progress in the development of CCD detectors for all wavelengths, they are victims of their high performance in the optical band, which generates a high noise compared to the desired UV band. By using solar-blind photocathodes our MCP detector avoids this problem and doesn't need extensive shielding or additional filters for a low signal-to-noise-ratio which, in combination with their fast readout, makes such a detector still leading in ultraviolet spectroscopy.

\subsection{Working principle}

Depending on the field of use, there are two concepts for MCP detectors, either a sealed detector with a semitransparent photocathode coated on an entrance window or an open-faced detector with an opaque photocathode directly on the micro channel plates. As the MCPs work with high voltage in the range of several thousand volts, they need to be operated under vacuum conditions with a pressure below $1 \times 10^{-5}$ mbar to avoid damage by accelerated particles. Therefore open-faced detectors only work in space, while sealed detectors can be operated everywhere, also in non-astronomical fields. Furthermore the photocathodes degrade under oxygen exposure, ${ }^{2}$ so they need to be stored under vacuum conditions (or inert gas), which complicates the use of open-faced detectors, as they need a sealed envelope until the detector is in space.

As shown in fig. 1 and fig. 2 any incoming photon that hits the photocathode, releases an electron which is accelerated towards the micro channel plates, where it creates an avalanche of additional electrons. Depending on the MCP configuration and applied voltage a gain factor of $1 \times 10^{5}$ to $1 \times 10^{7}$ is achieved. This electron cloud hits a charge sensitive anode and the centroid of the cloud will be calculated. Contingent on the sensitivity of the electronic readout system the amplification factor can be reduced, which enhances the lifetime of the micro channel plates, as only a material dependent amount of charge can be extracted out of each plate.

hermanutz@astro.uni-tuebingen.de

Space Telescopes and Instrumentation 2014: Ultraviolet to Gamma Ray, edited by Tadayuki Takahashi,

Jan-Willem A. den Herder, Mark Bautz, Proc. of SPIE Vol. 9144, 914434 · C 2014 SPIE

CCC code: $0277-786 X / 14 / \$ 18 \cdot$ doi: $10.1117 / 12.2054888$

Proc. of SPIE Vol. $9144914434-1$ 


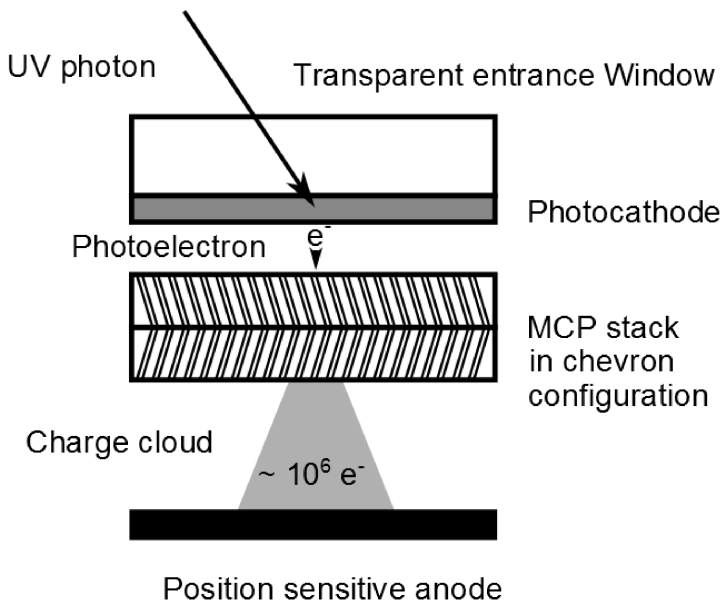

Figure 1. Detector principle in semitransparent mode ${ }^{3}$

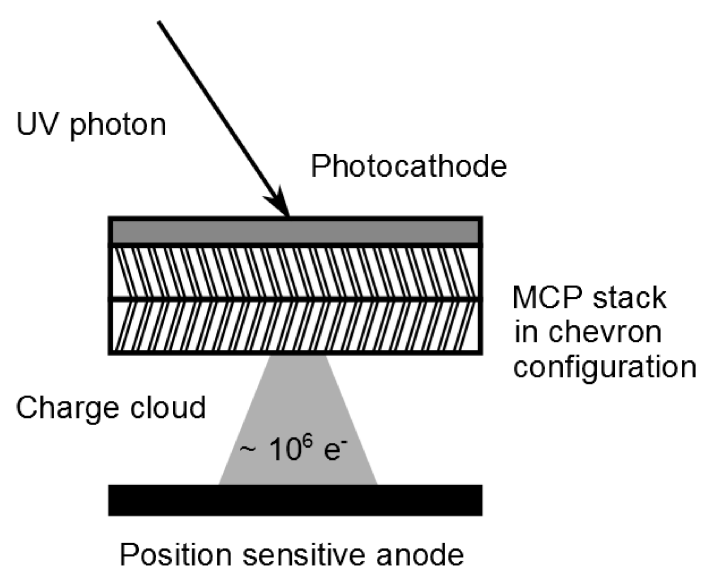

Figure 2. Detector principle in opaque mode

\subsection{Properties of MCP detectors}

The detector consists, as shown in the left part of fig. 3, from top to bottom, of four crucial parts:

1. the entrance window with the photocathode

2. the brazed detector body with the electrical contacts and its sealing

3. the micro channel plates

4. the cross-strip anode

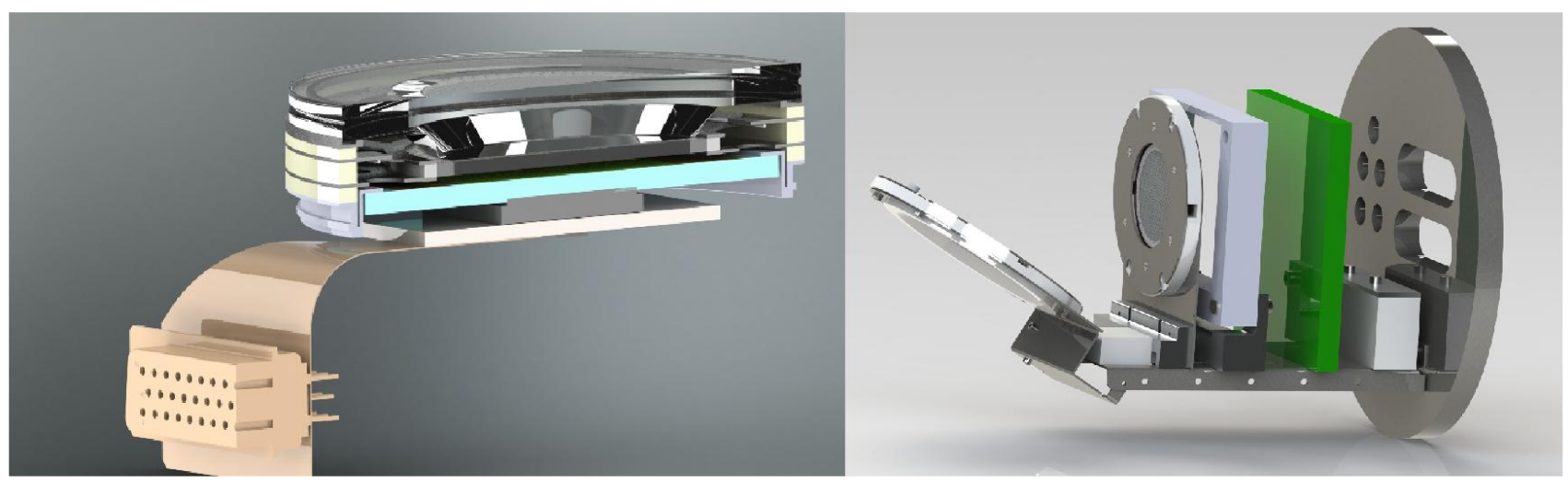

Figure 3. Left: Drawing of the detector layout including the anode readout through the Beetle chip. The detector is topped by an $80 \mathrm{~mm}$ (in diamater) entrance window and approximately $30 \mathrm{~mm}$ thick. Right: Drawing of the test setup for the MCP detector configuration and readout electronics

Each part offers various difficulties do deal with. The choice of the entrance window limits the wavelength range of the detector and determines the lower cut-off. Therefore we use magnesiumdifluoride windows, which offer UV transmission down to $150 \mathrm{~nm}$. Furthermore, the window material is also the substrate for the photocathode and needs to be compatible with the film growth mode of the used material. For example one can only grow a uniform (mono-crystalline) film of gallium nitride, if the lattice numbers of the substrate matches (like sapphire). In other cases a low sticking coefficient doesn't allow to grow a film at all. We consider to use 
either caesium activated gallium nitride or caesium-telluride as cathode material, depending on the performance of each material, whereat our facility is suited to make $\mathrm{Cs}_{2} \mathrm{Te}$ cathodes all by ourselves, or activate GaN samples from an external partner.

The detector body provides the electrical contacts for the micro channel plates, while being an insulator and needs to be leak tight for the whole lifetime of the detector in a region of $1 \times 10^{-10}$ mbar. Furthermore, the detector needs to survive a possible rocket launch to space and needs to provide mechanical and thermal stability. We decided for a brazed ceramic body with electrical contacts made of Kovar (an alloy consisting of $54 \%$ iron, $29 \%$ nickel and $17 \%$ cobalt which offers a similar thermal expansion as borosilicate glass and ceramics) to achieve a high thermal stability of the detector envelope and good leak tightness. The anode, all electrical contacts, the holders of the micro channel plates and the steering for the entrance window are brazed with the ceramics by the Swiss Federal Laboratories for Materials Science and Technology. The integration of the micro channel plates and the final sealing of the tube with the entrance window is done in Tübingen. The sealing will be done with a molten indium alloy ring, which melts, depending on the actual chemical composition, between $80^{\circ} \mathrm{C}$ to $150^{\circ} \mathrm{C}$. The ring will be placed and molten in a little flume within the steering whereon the window will be placed and pressed together.

We use standard $40 \mathrm{~mm}$ micro channel plates produced by Photonis, with a pore size of $10 \mu \mathrm{m}$ and a center-tocenter spacing of $12 \mu \mathrm{m}$ which leads to an open area ration of $55 \%$. The orientation of the micro channel plates, the distance between entrance window and plates and the distance between the plates and the anode determine the detector performance and therefore also their lifetime. The optimum configuration is still under investigation in a lab set-up (see left part of fig. 3), which allows a highly flexible simulation of the internal behavior of our detector and the readout (for more details see Pfeifer et al. ${ }^{4}$ ).

The fourth part of our detector is a cross-strip anode. There are three popular anode designs: the wedgeand-strip anode, the time-delay anode and the cross-strip anode. Each of these anodes uses a special pattern of conducting paths and therefore a special distribution of measured charge to calculate the position of the electron cloud. Whereas the wedge-and-strip anode only has four channels to readout and process, the needed charge and therefore also the needed gain factor is much higher compared to the other designs, which reduces the lifetime of the micro channel plates. The time-delay anode needs comparatively less charge, but instead of charge one measures the time-delay at four points, which require a precise electronics, as the time resolving power determines the spatial resolution of the anode. The cross-strip anode consists of two crossed layers of stripes, which are individually connected. If an electron cloud hits the cluster of stripes, one measures the charge on each single strip and based on the distribution the centre of the cloud can be calculated. Since the amount of stripes determines the spatial resolution of the anode, the capabilities of the electronics to readout channels limits the resolution of the anode. The cross-strip anode needs a similar gain factor as the time-delay anode, but offers a high spatial stability, as the position of each strip is well defined whereas the time-delay anode offers a lower dead time. As the detector is designed for a spectrograph, the higher spatial resolution is more important than a high temporal resolution offered by the time-delay-anode, therefore we decided for the cross-strip anode. Our electronics comprises the Beetle chip, ${ }^{5}$ which can pre-amplify, sample, and buffer 128 input channels and can multiplex the information to only four output channels which will be processed within a FPGA. Thus our cross-strip anode consists of $64 \times 64$ stripes.

At the current stages of developing our design is still highly flexible, as we investigate each part independent of the others. We can easily change the window material and try different photocathodes, change the amount, type or orientation of the micro channel plates by reconstructing the detector tube and could also switch between the cross-strip-anode or the time-delay anode, by changing the readout software and FPGA VHDL design. ${ }^{9}$ 


\section{CESIUM-TELLURIDE PHOTOCATHODES}

We currently investigate the possible use of $\mathrm{Cs}_{2} \mathrm{Te}$ as photocathode material, due to its band gap of $3.2 \mathrm{eV}$, which makes $\mathrm{Cs}_{2}$ Te only sensitive to radiation with a wavelength below $350 \mathrm{~nm} .{ }^{6}$ The basic configuration is about $8 \mathrm{~nm}$ to $10 \mathrm{~nm}$ of tellurium and additional $50 \mathrm{~nm}$ to $60 \mathrm{~nm}$ of caesium to obtain a total thickness of around $60 \mathrm{~nm}$ to $70 \mathrm{~nm}$ as calculated with Monte Carlo simulations by van Oerle ${ }^{7}$ to be the optimum thickness with a composition of Cs 2:1 Te. Basically, a thicker layer absorbs more photons, but reduces the amounts of escaping electrons. By variation of temperature, pressure, thickness and rate we search for an optimal setting in semi-transparent mode, with a high efficiency and long life time.

\subsection{Process and lab setup}

We use a high vacuum system consisting of two vessels, one to create the photocathodes on the window substrates and one vessel to seal and integrate the window onto the detector tube. The vessels are separated by a valve and are individually pumped down by a three stage pumping system, including an ion getter pump and a liquid nitrogen cooled titan sublimation pump, which allows us to reach a final pressure below $10 \times 10^{-10}$ mbar. Fig. 4 shows a schematic cross-section of the first vessel. We can store up to six substrates onto a rotating dish in the middle of the vessel. On the right side the photocathode coating takes place. We use an effusion cell to evaporate tellurium at temperatures between $250{ }^{\circ} \mathrm{C}$ to $300{ }^{\circ} \mathrm{C}$ and rates of $0.1 \AA \mathrm{s}^{-1}$ to $5 \AA \mathrm{s}^{-1}$. We use a micro balance rate controller to measure the rates and thickness during the evaporation and a $645 \mathrm{~nm}$ laser to measure the transmission of the substrate as cross check, as the tellurium growth at high rates and temperatures fails sometimes. Typically a thickness of $8 \mathrm{~nm}$ results in a $50 \%$ reduced transmission. During the evaporation the substrates are heated by radiation from the top. The caesium activation is done by Alvatec high purity caesium dispenser and is also monitored with the thickness monitor. During the activation the photocathode is illuminated with an UV-LED with emission at $256 \mathrm{~nm}$ and the photo current (or the dark current) is measured with a Keithley low current amperemeter (see fig. 6).

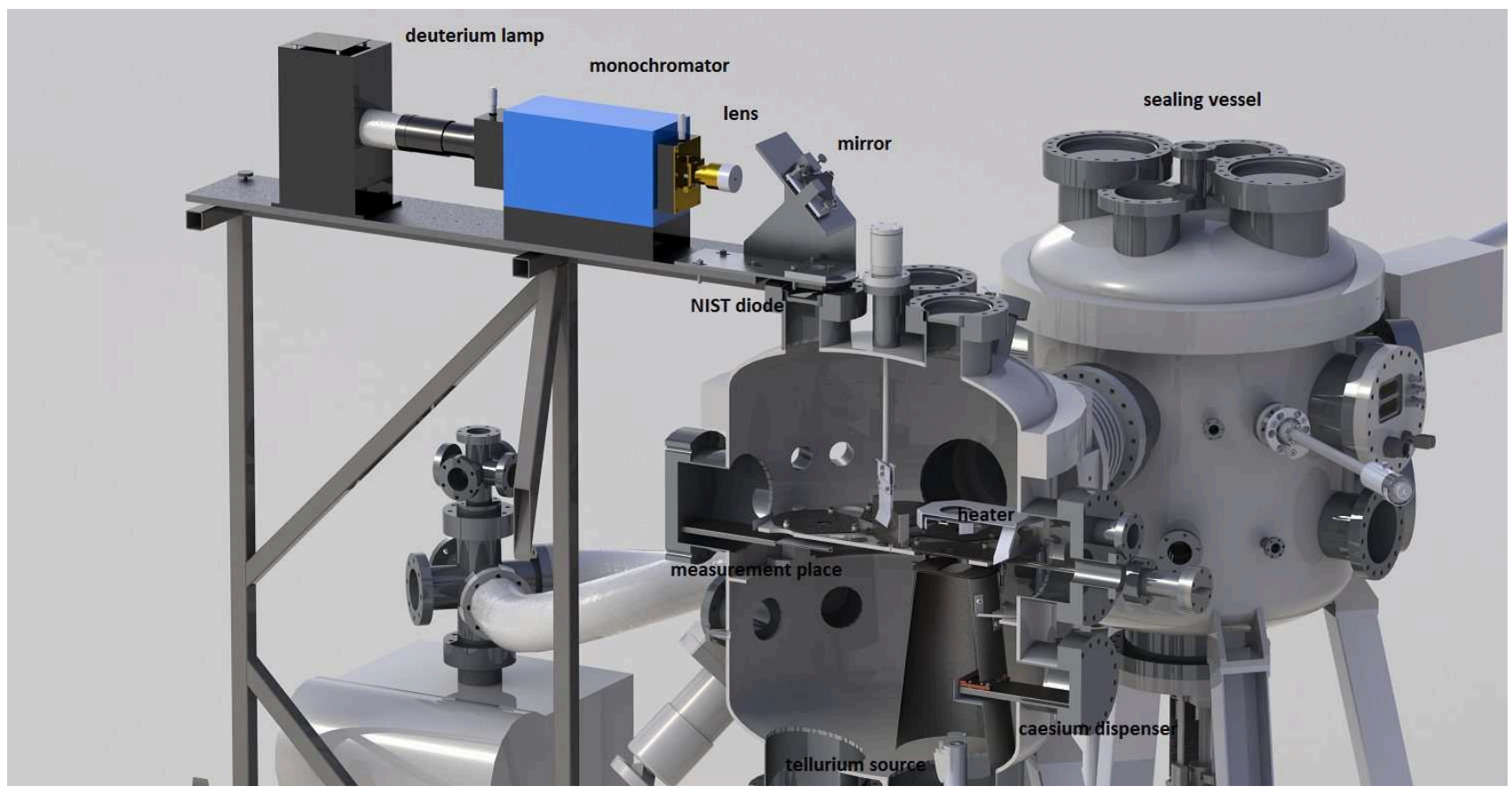

Figure 4. Cross-section drawing of the ultra high vacuum system used to produce photocathodes. On the right side of the vacuum vessel the substrates are heated and coated with tellurium and activated with caesium. On the left side the photocathodes can be qualified and measured. 


\subsection{Measurement of photocathode efficiency}

The possible use of a photocathode is determined by its wavelength dependent photo sensitivity, its spatial and its temporal stability. To qualify a photocathode we use a system of a deuterium lamp and a monochromator, combined with an NIST-calibrated diode (National Institute of Standard and Technology). The deuterium lamp emits at all wavelengths from $150 \mathrm{~nm}$ upwards, but due to our focussing optic consisting of a lens and a passive reflector (see fig. 4), there is no emittance of light until $183 \mathrm{~nm}$ and we do not expect any measurement up to $187 \mathrm{~nm}$ to be accurate, as the measured values are in the order of the dark current.

As it is impossible to realize an arrangement, which places the calibration diode inside the vacuum vessel, we need to correct our measurement by the transmission of the window, which was measured in advance. With a lens we aim to focus all light on the substrate, but as we can not make sure, that the UV light is solely on the cathode, all our measurements only give us a lower limit for the efficiency. We also measured the transmission of our substrates as we can change them and use other materials with higher performance in the ultraviolet like $\mathrm{MgF}_{2}$ like shown in fig. 5 .

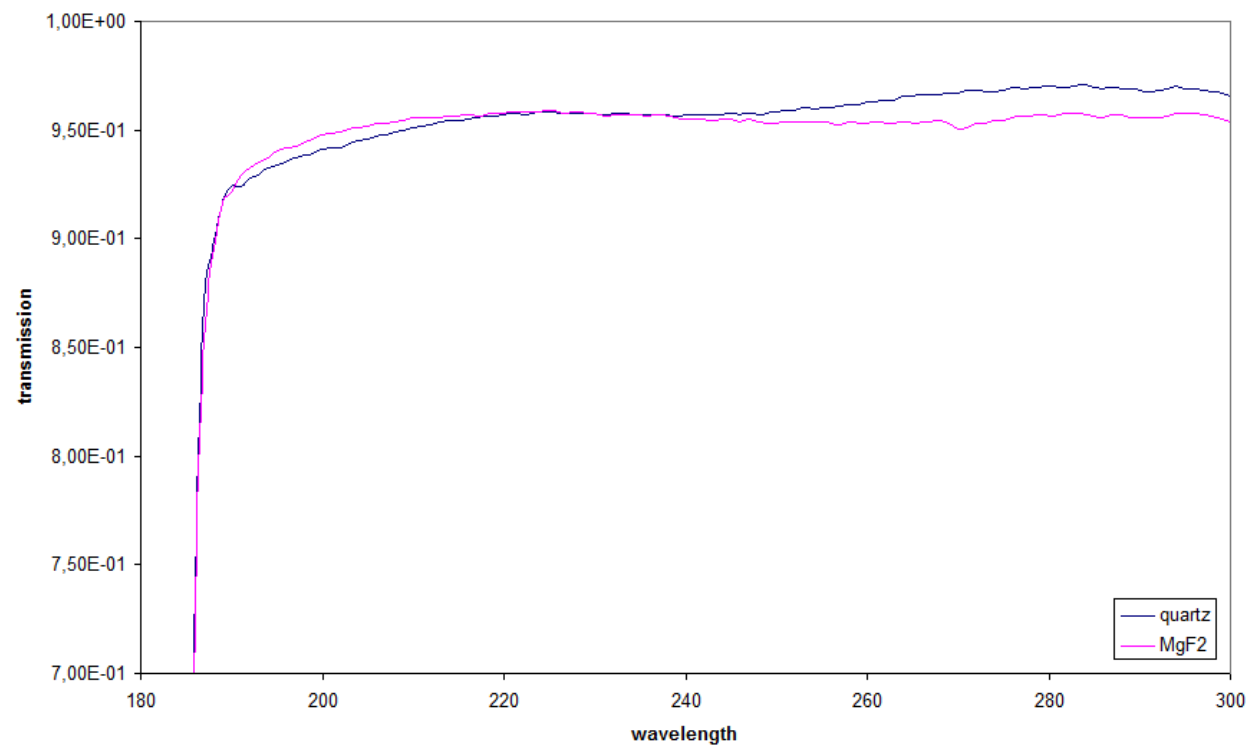

Figure 5. Transmission measurements of quartz substrates and $\mathrm{MgF}_{2}$ substrates with a calibrated NIST diode with our test ensemble. The lower cut-off at $187 \mathrm{~nm}$ is due to our optical system consisting of a focus lens an a refractive mirror and will be reduced in future by replacing the optical parts with better suited ones. One measurement run takes about $10 \mathrm{~min}$, while the lamp is given $2 \mathrm{~h}$ to stabilize itself and then provides a flux with less than $5 \%$ fluctuation per hour.

\subsection{Measurement of first photocathode}

Our system allows us to manipulate six parameters of the photocathode formation. The first parameter is the pressure in our vacuum vessel, which we aim to keep in the range below $1 \times 10^{-9}$ mbar during the whole process. The second parameter is the substrate temperature, which we adjust by radiation heating from top and needs to be at least $100^{\circ} \mathrm{C}$ to enhance the film formation. The last four parameters are the evaporation rate and the film thickness for each material, whereas the thickness is predicted by Monte Carlo simulations. The evaporation rates are limited by our devices and the optimum rates and their influence on the film growth and the resulting efficiency are under investigation. Until now we tested around 50 samples, whereas the first 30 samples where used for the unsuccessful attempt of cold photocathode production.

In fig. 7 our latest results is shown. It offers a peak efficiency of $14 \%$ at $190 \mathrm{~nm}$ and an overall efficiency of more than $4 \%$ under $270 \mathrm{~nm}$. The photocathodes are also solar blind, as the light response drops under $1 \%$ at $290 \mathrm{~nm}$. 


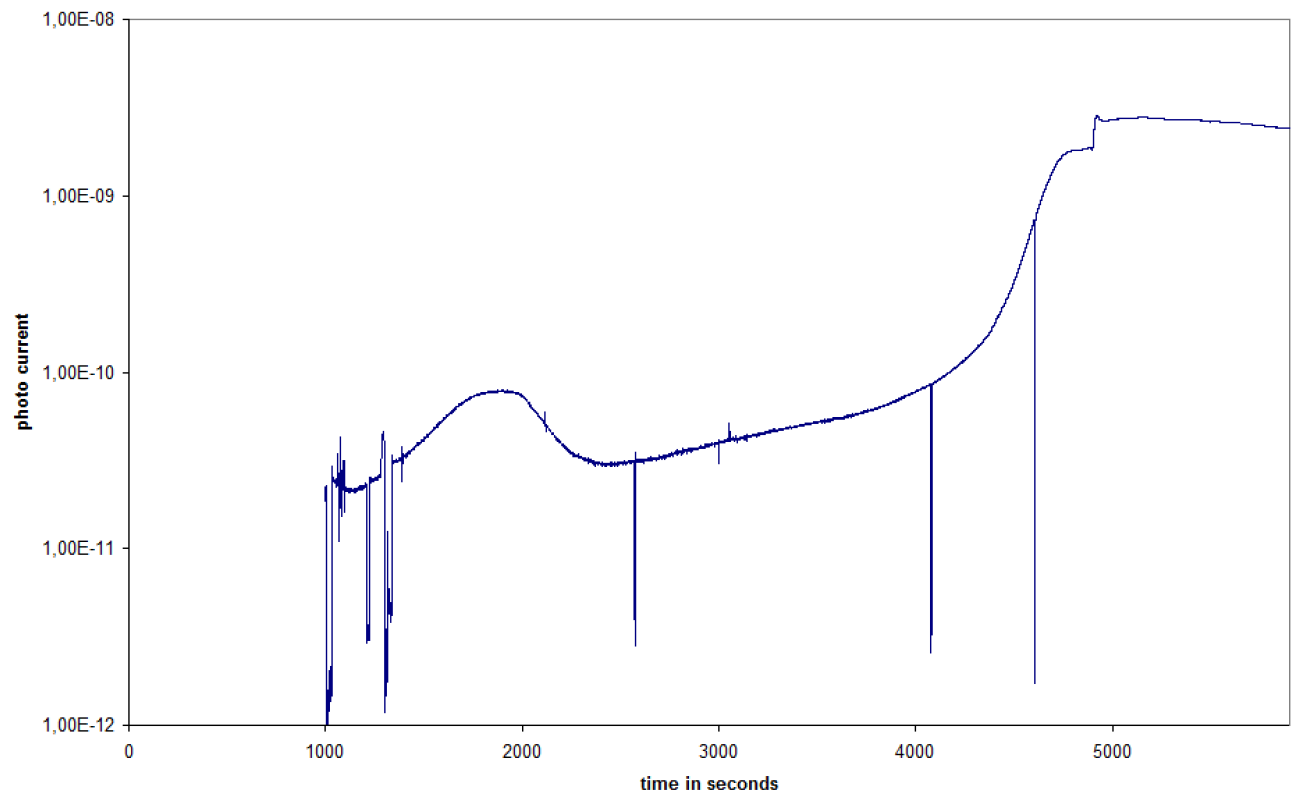

Figure 6. Photo current measurement of sample 46 illuminated with a $260 \mathrm{~nm}$-LED during the activation process. There are several stages of film formation which leads to a non linear characteristics of the photo response of the cathode. Activation started at time 1000s and ended at time 4902s which correlates with 65 minutes of caesium evaporation with an average rate of $0.1 \AA_{\mathrm{s}}^{-1}$. Right after stopping the caesium dispenser, the photo response increased sharply, due to an unkonwn effect. Most proberly it is a result of excess caesium which vaporises from the photocathode surface. The measured dark current is in the range of $2 \mathrm{pA}$ to $5 \mathrm{pA}$ which leads to a signal-to-noise-ratio in the order of 1000 .

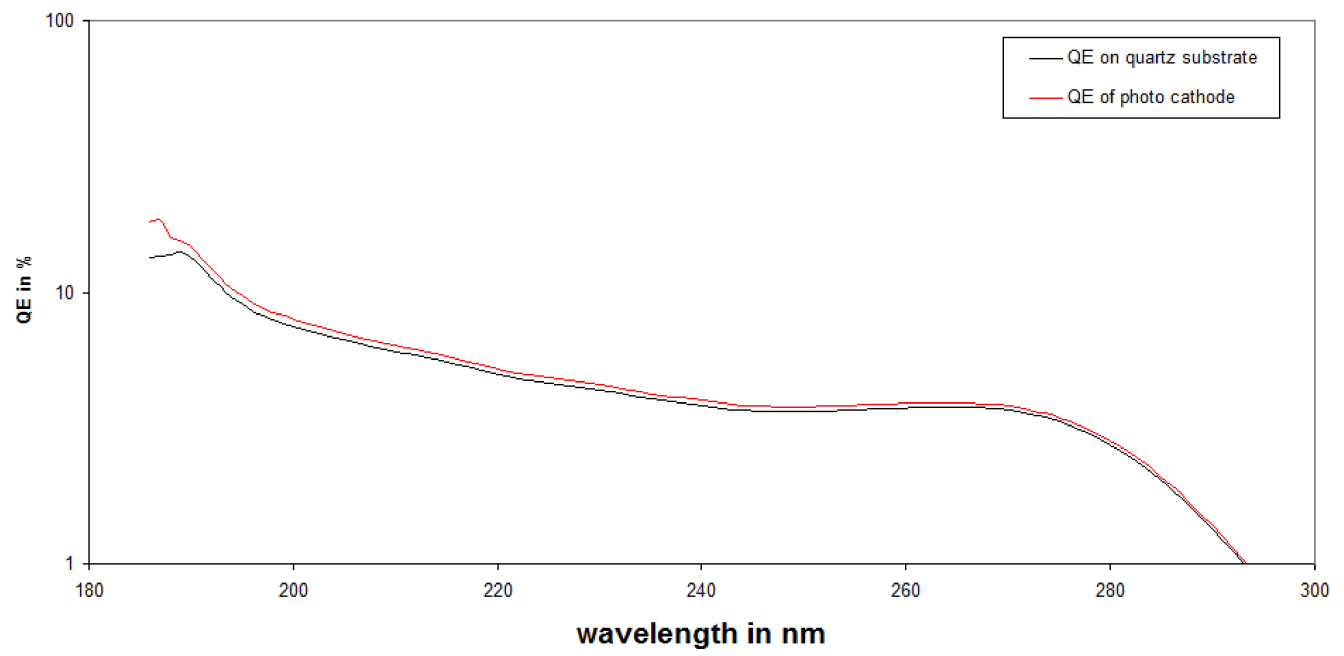

Figure 7. Measurement of working photocathode sample 48. As mentioned before there is a lower cut-off at $187 \mathrm{~nm}$. The black line is the measured total semitransparent efficiency on a quartz substrate, the red line the expected pure photocathode efficiency corrected for the transmission of the quartz substrate. In a next step we will measure a sealed photo diode without the optics to gain a total detector quantum efficiency and change the substrate material to $\mathrm{MgF}_{2}$, which provides a better transmission in the region below $200 \mathrm{~nm}$. 


\section{SUMMARY}

We are able to produce photocathodes with reasonable efficiency in the ultra violet range. In the next step we will seal them on top of the detector tubes to test the leak tightness and long term stability of the cathodes under working conditions. We also need to scale up the photocathode production process for the $80 \mathrm{~mm}$ entrance windows of the real detectors. In between the final steps of the micro channel plate optimizations are done, so that the final detector tubes can be produced.

\section{ACKNOWLEDGMENTS}

This work is funded by Deutsches Zentrum für Luft- und Raumfahrt (DLR) grant 50QT1003.

\section{REFERENCES}

1. Gomez de Castro, A.I., Pagano, I., Sachkov, M., et al.: In: Chavez, M., Bertone, E., Rosa-Gonzalez, D., Rodriguez-Merino, L.H. (eds.) "New Quests in Stellar Astrophysics. II. Ultraviolet Properties of Evolved Stellar Populations", p. 319. Springer, Berlin (2009)

2. di Bona, A., Sabary, F., Valeri, S., Michelato, P., Sertore, D., Suberlucq, G., "Auger and x-ray photoemission spectroscopy study on $\mathrm{Cs}_{2}$ Te photocathodes", [Journal of Applied Physics] (1996)

3. Diebold, S., Barnstedt, J., Kappelmann, N., Werner, K., et al., "MCP Detector Development for WSO-UV," in [Proceedings of SPIE Vol. 8443] (2012)

4. Pfeifer, M., Diebold, S., Barnstedt, J., Bauer, C., Werner, K., et al., "Low power readout electronics for a UV MCP detector with cross strip anode," in [Journal of Instrumentation vol. 9] (2014)

5. Löchner, S., "Development, Optimisation and Characterisation of a Radiation Hard Mixed-Signal Readout Chip for LHCb," (2006)

6. Taft, E., Apker, L., "Photoemission from Cesium and Rubidium Tellurides,"in [J. Opt. Soc. Am. vol 43, 81-83] (1953).

7. van Oerle, B., "A High Brightness Electron Beam for Free Electron Lasers" (1996)

8. Siegmund, O., et al., "Gallium nitride photocathode development for imaging detectors," in [Proceedings of SPIE Vol. 7021] (2008)

9. Pfeifer, M., Barnstedt, J., Kappelmann, N., Werner, K., et al., "Low-power readout electronics for micro channel plate detectors with cross-strip anodes," in [Proceedings of SPIE Vol. 8443] (2012) 